\title{
Thiopurine S-methyltransferase polymorphisms in acute lymphoblastic leukemia, inflammatory bowel disease and autoimmune disorders: influence on treatment response
}

This article was published in the following Dove Press journal:

Pharmacogenomics and Personalized Medicine

5 May 2017

Number of times this article has been viewed

\author{
Rachid Abaji' \\ Maja Krajinovic ${ }^{2}$ \\ 'Department of Pharmacology, \\ ${ }^{2}$ Departments of Pediatrics and \\ Pharmacology, CHU Sainte-Justine \\ Research Center, University of \\ Montreal, Montreal, QC, Canada
}

Correspondance: Maja Krajinovic Centre de recherche, $\mathrm{CHU}$ Sainte-Justine, 3175 Chemin de la Côte-Ste-Catherine, Montréal, QC H3T IC5, Canada

Tel +I 5I4345 493I Ext 6259 Email maja.krajinovic@umontreal.ca

\begin{abstract}
The thiopurine S-methyltransferase (TPMT) gene encodes for the TPMT enzyme that plays a crucial role in the metabolism of thiopurine drugs. Genetic polymorphisms in this gene can affect the activity of the TPMT enzyme and have been correlated with variability in response to treatment with thiopurines. Advances in the pharmacogenetics of TPMT allowed the development of dosing recommendations and treatment strategies to optimize and individualize prescribing thiopurine in an attempt to enhance treatment efficacy while minimizing toxicity. The influence of genetic polymorphisms in the TPMT gene on clinical outcome has been well-documented and replicated in many studies. In this review, we provide an overview of the evolution, results, conclusions and recommendations of selected studies that investigated the influence of TPMT pharmacogenetics on thiopurine treatment in acute lymphoblastic leukemia, inflammatory bowel disease and autoimmune disorders. We focus mainly on prospective studies that explored the impact of individualized TPMT-based dosing of thiopurines on clinical response. Together, these studies demonstrate the importance of preemptive TPMT genetic screening and subsequent dose adjustment in mitigating the toxicity associated with thiopurine treatment while maintaining treatment efficacy and favorable long-term outcomes. In addition, we briefly address the cost-effectiveness of this pharmacogenetics approach and its impact on clinical practice as well as the importance of recent breakthrough advances in sequencing and genotyping techniques in refining the TPMT genetic screening process.
\end{abstract}

Keywords: TPMT, pharmacogenetics, thiopurine, 6-mercaptopurine, azathioprine, ADRs.

\section{Introduction}

Thiopurine S-methyltransferase (TPMT) is an important cytoplasmic enzyme that catalyses the rate-limiting step in the metabolism of thiopurine drugs. It is coded by the TPMT gene and exerts its effect via S-adenosyl-L-methionine as the S-methyl donor and S-adenosyl-L-homocysteine as a by-product. ${ }^{1-3}$ Thiopurine drugs, mainly 6-mercaptopurine (6-MP), and its prodrug azathioprine (AZA), are implicated as antimetabolite cytotoxic and immunosuppressive agents in the treatment of malignancies such as acute lymphoblastic leukemia (ALL), inflammatory disorders like inflammatory bowel disease (IBD) and many autoimmune disorders, including rheumatoid arthritis (RA), systemic lupus erythematosus (SLE), autoimmune hepatitis (AIH) and generalized eczematous disorders. ${ }^{3-5}$ However, gastrointestinal disturbances (like nausea and vomiting), rashes, as well as more serious adverse drug reactions (ADRs) like bone marrow toxicity, hepatotoxicity and pancreatitis can lead to discontinuation of therapy in up to one-third of patients; ${ }^{6}$ these factors limit the use of these drugs. ${ }^{2}$ 
AZA is an inactive compound that must be converted into 6-MP via a glutathione-dependent process and both drugs eventually produce 6-thioguanine nucleotides (6-TGNs), a mechanism through which thiopurines exert both their cytotoxic and therapeutic effects. ${ }^{7,8}$ Numerous studies have demonstrated that the efficacy and toxicity of thiopurine drugs are correlated to the activity of the TPMT enzyme as this enzyme competes with xanthine-oxidase and hypoxanthine-guanine-phosphoribosyltransferase to determine the amount of 6-MP metabolized to 6-TGNs. ${ }^{1,7-10}$ 6-TGNs then either incorporate directly into DNA, which triggers delayed cytotoxicity, or they inhibit intracellular signaling pathways that ultimately promote cell death via apoptosis. ${ }^{11}$ Furthermore, 6-MP is also metabolized to methyl-thioinosine-monophosphate that provokes an additional cytotoxic effect by inhibiting de novo purine synthesis. ${ }^{12}$

Thioguanine (TG) is also a prodrug that belongs to the thiopurines family (2-amino-6-mercaptopurine) and is also partly metabolized by TPMT. Like AZA and 6-MP, it exerts its effect through mechanisms that involve the production of 6-TGNs, but they differ in the pathways implicated as depicted in Figure 1. However, due to its more pronounced toxicity profile and lack of additional benefit, its use became somewhat limited to the intensification phase of some antileukemia protocols. ${ }^{13}$

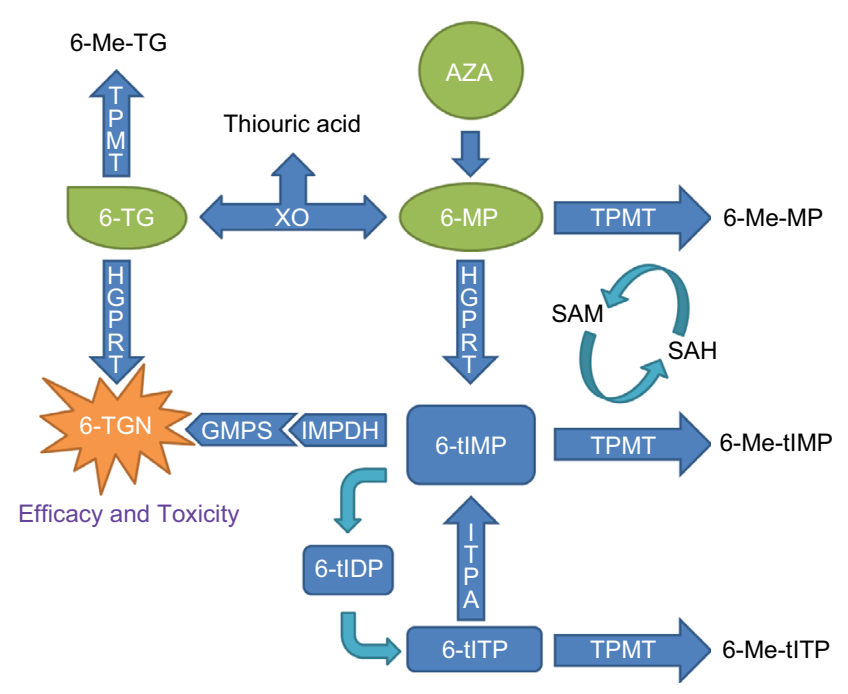

Figure I Metabolic pathways involved in the mechanism of action of thiopurines. Notes: This figure illustrated the pathways involved in the metabolism of Azathioprin, 6-mercaptopurine and thioguanine highlighting the genes/enzymes that can potentially affect the metabolism of these drugs.

Abbreviations: 6-MP, 6-mercaptopurine; 6-Me-MP, 6-methyl-mercaptopurine; 6-Me-TG, 6-methyl-thioguanine; 6-Me-tIMP, 6-methyl-thioinosine-monophosphate; 6-Me-tITP, 6-methyl-thioinosine-triphosphate; 6-TG, thioguanine; 6-TGN, 6-thioguanine nucleotides; 6-tIDP, 6-thio-inosine diphosphate; 6-tIMP, 6-thioinosine monophosphate; 6-tITP, 6-thio-inosine triphosphate; AZA, azathioprine; GMPS, guanosine monophosphatase synthetase; HGPRT, hypoxanthine guanine phosphoribosyl transferase; IMPDH, inosine monophosphate dehydrogenase; ITPA, inosine triphosphate pyrophosphatase; SAH, S-adenosyl-L-homocysteine; SAM, S-adenosyl-L-methionine; TPMT, thiopurine S-methyltransferase; $\mathrm{XO}$, xanthine oxidase.
TPMT deficiency was first described around 3 decades ago and it is currently well established that homozygous or compound heterozygous carriers of TPMT-deficient alleles have a significantly higher risk of early severe myelosuppression than patients homozygous for the wild-type. ${ }^{14,15}$ Patients with absent or reduced TPMT activity accumulate high doses of 6-TGNs, resulting in thiopurine-induced myelotoxicity that is characterized by early onset of severe neutropenia when such patients are treated with standard doses of thiopurine drugs. This toxicity is particularly evident in patients carrying two nonfunctional alleles and requires treatment cessation or dose adjustment. ${ }^{1,5,11,16-21}$ Bone marrow suppression has been linked to higher cumulative incidence of infections, mortality and death. ${ }^{5,22,23}$ Conversely, myelosuppression can be induced by a number of factors independent of TPMT in individuals taking thiopurines, that is co-medications, viral infections, underlying disease and idiosyncratic reactions, ${ }^{24}$ as well as genetic polymorphisms in genes other than TPMT encoding enzymes involved in thiopurines metabolism like inosine triphosphate pyrophosphatase (ITPA) ${ }^{25}$ and Nudix hydrolase $15(N U D 15)^{26}$ genes. On the other end of the spectrum, some studies indicated that high TPMT activity has been linked to poor treatment response and that an elevated dose is needed in order to achieve therapeutic effect. ${ }^{27,28}$

\section{TPMT pharmacogenetics}

Enzymatic activity of TPMT can be indirectly assessed through red blood cell enzyme activity assay (phenotype) or can be inferred from the genetic profile of the white blood cells. ${ }^{1,11,29}$ Genetic polymorphisms in the TPMT gene can affect the enzymatic activity of TPMT and have been studied extensively. To date, over 38 variant alleles have been identified. ${ }^{2,15,30-32}$ They have been correlated with variability in response to thiopurine drugs, which provides an important example of the clinical importance of pharmacogenetics. Nonetheless, only a few of these polymorphisms are considered in clinical settings that can identify the most frequently reduced-activity TPMT alleles and account for $\geq 95 \%$ of variant TPMT alleles. ${ }^{12,18,31-33}$ The wild-type allele is defined as $T P M T^{*} 1$. The mutant $T P M T^{*} 2$ allele is defined by the $\mathrm{G} 238 \mathrm{C}$ transversion whereas the $T P M T^{*} 3$ family alleles are defined by the G460A and A719G transitions (i.e., $T P M T^{*} 3 A[\mathrm{G} 460 \mathrm{~A}$ and $\mathrm{A} 719 \mathrm{G}], T P M T^{*} 3 B[\mathrm{G} 460 \mathrm{~A}]$ and $\left.T P M T^{*} 3 C[\mathrm{~A} 719 \mathrm{G}]\right)^{12,18,31-33}$ The prevalence of $T P M T$ variants is much higher among Caucasians $(8.1 \%-10.1 \%)$ than Asian populations $(2.3 \%-4.2 \%)^{15}$ and it is well established that $T P M T^{*} 3 A$ is the most prevalent mutant allele in Caucasians, making up to ( $85 \%)$ of all observed mutant alleles, ${ }^{14,18}$ 
while $T P M T^{*} 3 C$ is the most frequently found allele in African and Southeast Asian populations. ${ }^{14,34}$

Other than variants in the coding region of TPMT, it is being increasingly acknowledged that variants in the noncoding regions such as the TPMT-promoter and introns can also affect the activity of the TPMT enzyme, possibly by influencing the transcription of its gene. ${ }^{3}$ One well-studied example of such polymorphisms is the variable number of tandem repeats (VNTR) region, which is a rare microsatellite region in the TPMT gene promoter. Interestingly, studies have shown that the architecture of this region can modulate TPMT transcription and possibly enzyme activity. For example, higher TPMT promoter activity was shown to be associated with a region that contains five or seven GCC repeats rather than six. Thus, studies suggest the use of VNTR architecture as a pharmacogenomic biomarker to refine the TPMT genetic screening process currently used prior to the introduction of thiopurine therapy to enhance the treatment outcome in ALL. ${ }^{3}$ However, contrary to the results of ALL studies, the expression of the TPMT gene seems to decrease in IBD patients treated with thiopurine drugs and thus VNTR genotype cannot predict the TPMT activity, which seems to be influenced by the treated condition, the protocol used and the concomitant administration of other drugs. ${ }^{3}$

Across all ethnic groups, $\sim 1$ in 300 individuals are homozygous (or compound heterozygous) for a mutant TPMT allele and have very low or absent TPMT activity while around $4 \%-11 \%$ of individuals are heterozygous and are generally considered to have intermediate enzymatic activity. ${ }^{1,18,31}$ Nonetheless, such genotype-based classification is not always representative of the actual state of enzymatic activity. In literature, conflicting data were obtained by studies that addressed the concordance between the genetic and phenotypic tests, as results ranged from $100 \%$ or almost perfect match in the majority of studies to as low as $77 \%$ concordance in a few of them. ${ }^{1,3,9-11,19,33,35-38}$ This discordance was particularly observed in patients with intermediate activity in whom the estimated probability of obtaining matching results varied from $70 \%$ to $86 \% .{ }^{11,33,36,39}$

Many factors influence TPMT enzyme activity and eventually affect this genotype-phenotype concordance such as the age and gender of the patient, coadministration of drugs that could potentially interfere with the disease condition or TPMT activity (e.g., methotrexate), ${ }^{40}$ levels of TPMT cofactor S-adenosyl-methionine, ${ }^{41}$ recent blood transfusion, ${ }^{42}$ life span of red blood cells,${ }^{43}$ as well as untested rare or novel variants in the coding and regulatory regions of the TPMT gene (e.g., TPMT*38 and the VNTR architecture). ${ }^{3,37,44}$ Furthermore, interethnic variability in the TPMT enzymatic activity levels have been observed in people of Afro-Caribbean descent having lower activity than Caucasians and South Asians. ${ }^{1,45,46}$ Taken together, there is always a risk of misclassifying patients if the decision was based on only one of the two abovementioned methods, but it is also unreasonable to perform both tests for all patients. A recent randomized clinical trial (RCT) concluded that there was no advantage or disadvantage of TPMT genotyping compared with phenotyping, ${ }^{16}$ whereas a more recent study concluded that genotyping was superior to phenotyping and should be considered as the primary choice for pretreatment evaluation of TPMT function. ${ }^{35}$ Nonetheless, phenotype testing supplemented by genotyping can be a useful strategy in specific circumstances (e.g., after recent blood transfusion and for confirmation of intermediate activity in known highrisk patients). ${ }^{16}$

Recent advances in the pharmacogenetics of TPMT have allowed for the development of dosing recommendations and treatment strategies to optimize and individualize therapy with thiopurines to obtain maximum treatment benefit with minimal toxicity ${ }^{47}$ However, the implementation of pharmacogenetic tests in clinical practice is still somewhat limited due to the lack of robust evidence stemming directly from large-scale RCTs and proving the clinical utility of such strategy. ${ }^{1,16,22}$ Nonetheless, given the indisputable influence of pharmacogenetics on TPMT activity and the seriousness of thiopurines-induced toxicities, particularly myelosuppression, several regulatory agencies and clinical guidelines such as the US Food and Drug Administration, British National Formulary and Clinical Pharmacogenetics Implementation Consortium (CPIC) recommend pretreatment TPMT activity testing either by genotyping or phenotyping. ${ }^{11,47}$ In general, most guidelines suggest that the initial dose of thiopurines should be reduced to $10 \%$ of the standard dose when administered to homozygous carriers of TPMT-deficient alleles, as well as a reduction in administration frequency. For heterozygous patients, the recommendation differs slightly depending on the type of thiopurine used, as CPIC guidelines suggest an initial dose of AZA and 6-MP that is $30 \%-70 \%$ of the standard protocol dose while the recommendation for TG is $30 \%-50 \%$ of that dose. ${ }^{47}$

As new data is being continuously generated by RCTs and studies about the long-term outcome of previous treatment protocols, the strength of the clinical evidence should be constantly revised and the recommendations of the guidelines should be reevaluated and modified when deemed necessary. 
In this review, we provide an overview of the evolution, results, conclusions and recommendations of studies that investigated the influence of TPMT pharmacogenetics on clinical response to thiopurines in ALL, IBD and autoimmune disorders.

\section{Acute lymphoblastic leukemia}

Childhood ALL is the most frequent pediatric cancer. The survival rate currently exceeds $85 \%$ in favorable settings. 6-MP is coadministered with methotrexate as key components in the maintenance therapy for pediatric ALL and their use is associated with significant reduction in disease relapse. ${ }^{33}$

An early study reported that TPMT activity was significantly higher in blood samples of ALL patients on long-term 6-MP treatment compared with controls. ${ }^{48}$ They also noted a relationship between low TPMT activity and the risk of developing severe myelosuppression in patients treated with thiopurine drugs, plausibly due to elevated 6-TGN concentrations. ${ }^{48,49}$ Others reported that higher TPMT activity was linked to an elevated risk of relapse..$^{50}$ These findings led to the suggestion that genetic screening of TPMT activity could play a role in influencing treatment response to childhood ALL. ${ }^{48,50}$ Indeed, one study of childhood ALL suggested that prospectively screening for major TPMT coding region polymorphisms followed by selective administration of an initially reduced dose of 6-MP to heterozygous patients and a subsequent gradual increase to a target range of blood cell count allowed these patients to eventually achieve the full drug dose without experiencing any toxicity. ${ }^{51}$ Many clinical trials have investigated the impact of TPMT gene polymorphisms on treatment outcome, with most of them demonstrating the benefit of preemptive TPMT screening, but results were somewhat inconsistent (Table 1).

The Total Therapy Study XII explored the impact of 6-MP dose reduction from the standard protocol dose to a maximum tolerable dose subsequently to the development of myelosuppression and investigated the association between the maximum tolerable doses and TPMT genotypes in a total of 188 patients. The results showed that TPMT genotype was an important predictor of 6-MP toxicity in ALL patients, as the cumulative incidence of dose reduction or treatment interruption was significantly different across the 3 groups $(P<0.001)$; the results also showed that wild-type patients had the lowest incidence $(7 \%)$ followed by heterozygous carriers $(35 \%)$ and homozygous carriers of TPMT-deficient alleles $(100 \%) .{ }^{10,23}$ They also concluded that administering lower doses of 6-MP in these patients was successful in maintaining adequately high levels of 6-TGN while allowing the administration of other agents at full protocol doses. ${ }^{10}$ Furthermore, the investigators pointed out that the reduced activity patients tended to improve event-free survival (EFS) compared with wild-type patients $(P=0.096)$ and that higher dose intensity of 6-MP was the most significant predictor of that outcome $(P=0.020) .{ }^{23}$ However, the authors also observed a nonsignificant trend for patients with low TPMT activity to have higher incidence and shorter onset of secondary acute myeloid leukemia as well as higher cumulative incidence of brain tumors when compared with patients with wild-type. ${ }^{52,53}$ In their later trial, Total Therapy Study XIIIB, which included 247 patients and pioneered the implementation of pharmacogenetics in leukemia therapy, they continued to administer a standard initial dose of 6-MP at the start of the continuation therapy but then selectively decreased the dose when deemed necessary based on a strategy that involved up-front knowledge of TPMT status combined with clinical tolerance and measurement of thiopurine metabolite levels. ${ }^{54,55}$ They eventually reported that TPMT genotype was not associated with the risk of hematologic relapse and that the long-term outcome showed no association with TPMT status (5-year cumulative incidences of $13.2 \% \pm 2.3 \%$ and $6.7 \% \pm 6.7 \%$ for wild-type and low-activity genotypes, respectively; $P=0.46$ ), further confirming that considering pharmacogenetics of TPMT for dose adjustment of 6-MP dosage in ALL can help to reduce treatment-associated toxicity while not compromising its efficacy. ${ }^{54-57}$

In the NOPHO-ALL-92 study of The Nordic Society of Paediatric Haematology and Oncology, a higher risk of relapse was observed in patients homozygous for wildtype $(P=0.02)$ and/or high TPMT activity $(P=0.002) .{ }^{36,58,59}$ However, the authors also observed that patients with low TPMT activity, although at lower risk of relapse, had a higher risk of developing second myeloid neoplasms (SMN) associated with high levels of 6-TGN and methylated metabolites, probably leading to DNA damage and subsequent malignancies. The authors believe that this theory explains why low TPMT activity patients did not have a superior overall survival (OS) compared with those with wild-type activity $(P=0.82)$ despite their lower risk of relapse. ${ }^{38,60}$ These observations, together with the ones from the Total Therapy Study XII, led the NOPHO to adopt TPMT genotype-dependent initial dosing of 6-MP in their later protocols, ALL-2000 and ALL-2008..$^{36,58}$ Indeed, the longterm survival results from the ALL-2000 trial indicate that selecting the initial 6-MP dose based on TPMT genotype did reduce the risk of SMN in heterozygous patients, but at the expense of an increased risk of relapse. This explains 


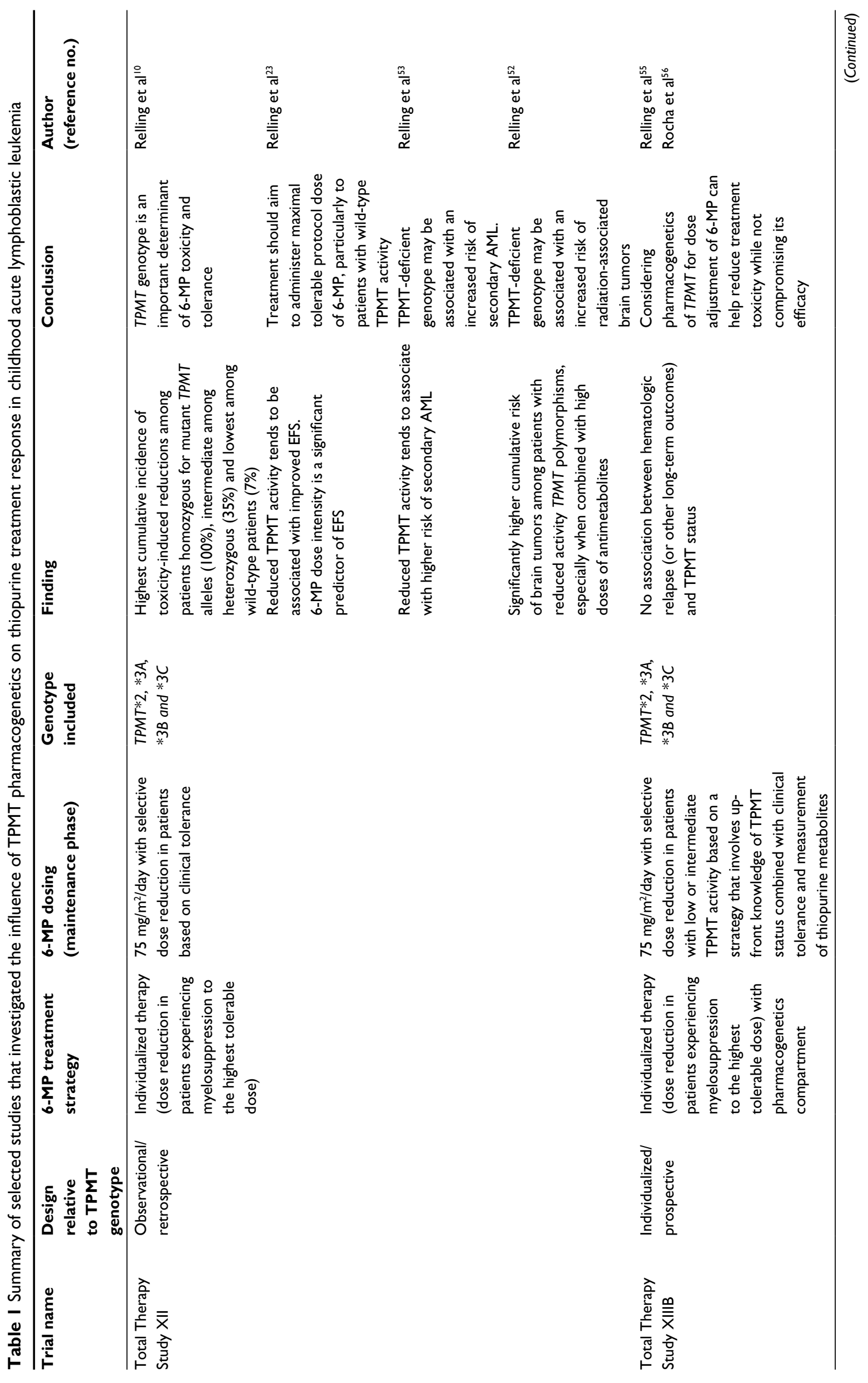




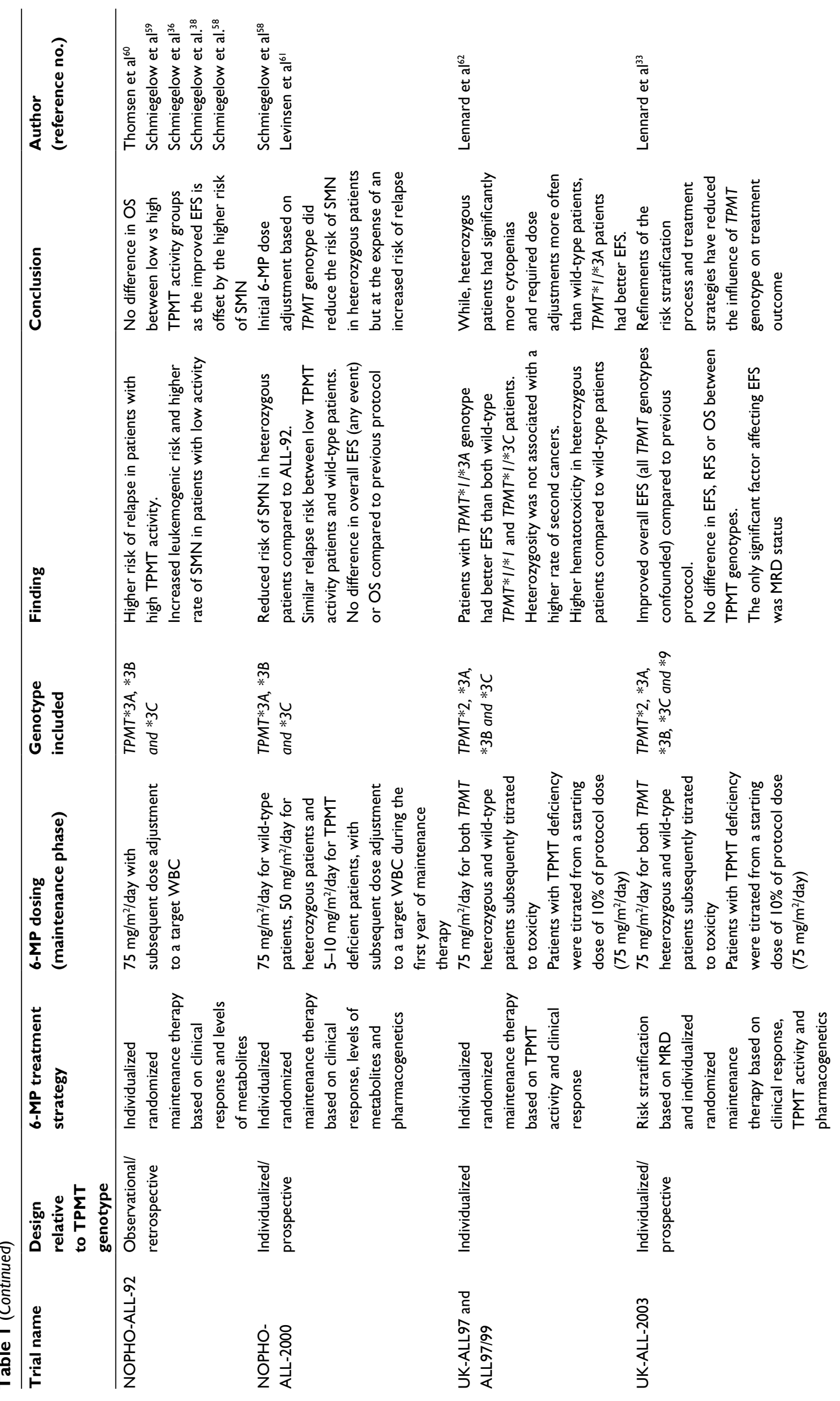



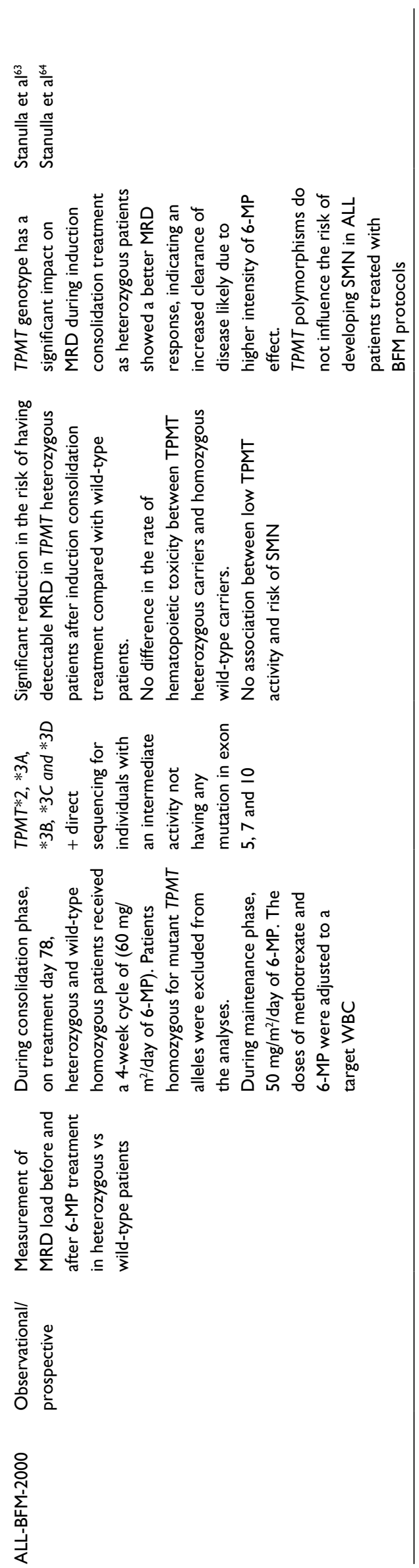

why although a slight nonsignificant improvement in EFS was achieved by the new protocol, it had no difference in overall EFS or OS from its predecessor (5-year results: NOPHO-ALL-92: $E F S=77.4 \% \pm 1.0 \%$, OS $=87.6 \% \pm 0.8 \%$, $\mathrm{n}=1654$; and NOPHO-ALL-2000: $\mathrm{EFS}=79.4 \% \pm 1.5 \%$ and OS $=89.1 \% \pm 1.1 \%, \mathrm{n}=1023) .{ }^{58,61}$

In the UK, ALL97and ALL97/99 trials, wild-type and heterozygous patients on the 6-MP arm initially received full dose of the drug, which was later adjusted to clinical hematologic toxicity whereas TPMT-deficient patients received $10 \%$ of the dose also adjusted for toxicity. While investigators observed a finding similar to the aforementioned studies in that patients with the $T P M T^{*} 1 / * 3 A$ genotype $(\mathrm{n}=99$, EFS=88\%) had better outcome at 5 years compared with TPMT wild-type patients $(\mathrm{n}=1206, \mathrm{EFS}=80 \% ; P=0.05)$, paradoxically, patients with the TPMT*1/*3C genotype also had lower EFS than those with $* 1 / * 3 A$ genotype $(\mathrm{n}=17, \mathrm{EFS}=53 \% ; P=0.002)$. Furthermore, patients with heterozygous genotypes were found to experience more myelosuppression, accumulated higher 6-TGN concentrations and required dose reduction more frequently. However, no association between the risk of secondary malignancy and TPMT genotype was found. . $13,33,62$ In their subsequent trial, ALL-2003, which used minimal residual disease (MRD) to guide risk stratification and treatment intensity, the protocol prospectively observed the influence of $T P M T$ genotype on treatment outcome by applying pretreatment genetic screening of the most common TPMT polymorphisms to 2387 of the study patients. The dosing regimen for 6-MP was similar to that of ALL97 in the sense that TPMT-deficient patients received $10 \%$ of the dose while the others received a standard dose subsequently adjusted according to a target cell count. The results showed that overall EFS - all TPMT genotypes confounded - was significantly higher than that of the previous protocol, which was attributed to the improved survival in the TPMT wild-type and $T P M T^{*} 1 /{ }^{*} 3 C$ genotype groups (EFS at 5 years $=88 \%, 88 \%$ and $94 \%$ for $T P M T$ wildtype, ${ }^{*} 1 / * 3 A$ and $* 1 / * 3 C$, respectively). However, within this protocol, no significant differences in OS, EFS or relapse-free survival were observed with respect to TPMT genotypes. Thus, it was concluded that the improved risk-adapted protocol had reduced the influence of TPMT genotypes on treatment outcome and that the only factor that affected the outcome was MRD. Furthermore, there was no difference in survival within each MRD risk group with respect to TPMT genotypes. ${ }^{33}$ Overall, the cumulative experience of the many UK ALL trials led to mandating preemptive TPMT screening for all children and young adults who start the ALL-2011 trial protocol. ${ }^{11}$ 
In the Berlin-Frankfurt-Münster-2000 (BFM-2000) trial, the 6-MP dose was reduced by 10 -fold from the standard starting dose for TPMT-deficient patients, but no dose adjustment was carried for heterozygous carriers who were given doses equal to the homozygous carriers of the wild-type. The investigators assessed the genotypes of 814 patients and used MRD for risk-stratification. The results showed no difference in the rate of hematopoietic toxicity between TPMT heterozygous variant carriers and homozygous wild-type carriers or between TPMT status and the risk of developing secondary cancers. Interestingly, it was observed that TPMT genotype had a significant impact on MRD during induction consolidation treatment as heterozygous patients had better MRD response (2.9-fold reduction), indicating an increased clearance of disease likely due to higher intensity of 6-MP effect (Relative risk $=0.34 ; 95 \%$ confidence interval $[\mathrm{CI}], 0.13-0.86 ; P=0.02) .{ }^{63,64}$

In summary, all these trials demonstrate the importance of preemptive TPMT genetic screening and subsequent dose adjustment in mitigating the toxicity associated with thiopurine treatment while maintaining, if not enhancing, treatment efficacy and favorable long-term outcome.

\section{Inflammatory bowel disease}

IBD is a polygenic chronic, relapsing and remitting disease of the gastrointestinal tract that can be divided into two major clinical subtypes, Crohn's disease and ulcerative colitis., ${ }^{2,65}$ Thiopurines, particularly AZA, are proven effective in inducing and maintaining long-term remission in IBD patients. ${ }^{5}$ More than $20 \%$ of patients experience severe ADRs that lead to dose modification, treatment interruption or cessation. ${ }^{66,67}$ Bone marrow toxicity represented by leukopenia is one of the most serious thiopurine-related ADRs. Many studies investigated the influence of TPMT genotype on the efficacy and toxicity of thiopurines and most suggested a significant impact on clinical response (Table 2).

A systematic review followed by a meta-analysis that eventually combined the results of 47 studies that investigated the risk of myelosuppression with respect to intermediate TPMT activity demonstrated a 4.19-fold increase in oddratio of leukopenia (95\% CI: 3.20-5.48) in IBD patients with reduced TPMT activity compared with wild-type. One critic of this meta-analysis is that it combined rather smaller studies with sample sizes of $<100$ patients in most cases and the majority having retrospective cohort designs. However, in a sub-analysis of this study that combined 834 patients coming only from the 11 studies that had a prospective cohort design, the significant association of reduced TPMT activity with the risk of myelosuppression had an odd-ratio of 4.3 (95\% CI: $2.53-7.29) .{ }^{1}$ Among these prospective studies, an observational study with preemptive TPMT genetic testing for all patients and a relatively large sample size of 207 participants found that heterozygous TPMT genotype strongly predicted treatment withdrawal due to early-onset of ADRs following a conventional fixed-dosing regimen (79\% vs $35 \%$ in heterozygous and wild-type, respectively; $P<0.001)$. They highlighted that gastric intolerance (GI) was the most frequent reason for withdrawal among this group of patients and that myelotoxicity and GI occurred significantly more frequently among heterozygous (26\% and $37 \%$, respectively) than wild-type patients ( $0.5 \%$ and $7 \%$, respectively). Interestingly, they had a $100 \%$ concordance of genotype to phenotype activity and found that TPMT activity was strongly predictive of clinical response as it was significantly higher in nonresponders. ${ }^{68}$ Other prospective studies with preemptive TPMT genetic screening component reported that overall thiopurine-related ADRs were significantly more common among patients with low-to-intermediate TPMT activity when doses were not adjusted; ${ }^{67}$ particularly myelotoxicity, which was more profound in TPMT-deficient genotype. ${ }^{67,69}$

Two independent meta-analysis further investigated the impact of pharmacogenetics on treatment response by exclusively combining studies (14 and 9 studies, respectively) that investigated the association between TPMT polymorphisms and ADRs in IBD patients, regardless of the study design (i.e., cross-sectional cohort, prospective cohort and case-control studies). ${ }^{2,65}$ They involved 2206 and 1309 patients respectively, and both concluded that TPMT polymorphisms were significantly associated with thiopurine-induced overall ADRs and bone marrow toxicity (around 3- and 6-fold increase in the odd-ratios, respectively) but not with hepatotoxicity, pancreatitis, flu-like symptoms, GI or skin reactions., ${ }^{2,65}$

The TPMT: Azathioprine Response to Genotyping and Enzyme Testing (TARGET) trial is a pragmatic RCT that prospectively investigated the impact of genotype-guided initial dosing of AZA followed by upward-titration to the maximum tolerable dose of the full protocol dose compared to no genotyping and full standard dose administration to all participants. It included 333 patients with inflammatory diseases and the primary aim was to see if this strategy would result in a significant reduction in the rate of ADRs-induced treatment cessation. No differences were found between the conventional and pharmacogenetics arms with respect to the frequency of treatment interruption due to ADRs (frequency: $27.7 \%$ vs $28.8 \%$; odds ratio [OR]: 1.1 ; 95\% CI: $0.66-1.8$; $P=0.74)$. On the other hand, the study did not find any difference in the rate of remission between the intervention 
Table 2 Summary of selected studies which investigated the influence of TPMT pharmacogenetics on thiopurine treatment response in inflammatory bowel disease

\begin{tabular}{|c|c|c|c|c|}
\hline $\begin{array}{l}\text { Author } \\
\text { (reference no.) }\end{array}$ & $\begin{array}{l}\text { Design relative } \\
\text { to TPMT } \\
\text { genotype }\end{array}$ & Treatment strategy & $\begin{array}{l}\text { Genotypes } \\
\text { included }\end{array}$ & Findings and conclusion \\
\hline Derijks et al ${ }^{69}$ & Observational & $\begin{array}{l}\text { 6-MP as a single oral } 50 \mathrm{mg} \\
\text { evening dose }\end{array}$ & $\begin{array}{l}\text { TPMT*2, *3A, } \\
* 3 B \text { and } * 3 C\end{array}$ & $\begin{array}{l}\text { TPMT genotype correlated with 6-TGN } \\
\text { concentrations. Patients with mutant alleles have } \\
\text { higher risk of developing leukopenia }\end{array}$ \\
\hline Hindrof et $a^{67}$ & Observational & $\begin{array}{l}\text { Dose escalation schedule } \\
\text { to the target dose of } \\
(2.5 \mathrm{mg} / \mathrm{kg}) \text { for azathioprine } \\
\text { and }(1.25 \mathrm{mg} / \mathrm{kg}) \text { for } 6-\mathrm{MP} \text { by } \\
\text { week } 3\end{array}$ & $\begin{array}{l}\text { TPMT*2, *3A, } \\
* 3 B, * 3 C, 3 D \\
* 4, * 5, * 6, * 7, * 8, \\
* 10, * 14 \text { and } * 15\end{array}$ & $\begin{array}{l}\text { Overall, thiopurine-related ADRs were } \\
\text { significantly more common among patients with } \\
\text { low-to-intermediate TPMT activity; particularly } \\
\text { myelotoxicity in TPMT-deficient patients }\end{array}$ \\
\hline Ansari et al ${ }^{68}$ & Observational & $\begin{array}{l}\text { AZA was started at } \\
2 \mathrm{mg} / \mathrm{kg} \text { daily and without dose } \\
\text { alteration }\end{array}$ & $\begin{array}{l}\text { TPMT*3A, *3B } \\
\text { and } * 3 C\end{array}$ & $\begin{array}{l}\text { Heterozygous TPMT genotype strongly predicts } \\
\text { treatment withdrawal due to early-onset of ADRs. } \\
\text { Gastric intolerance was the most frequent reason } \\
\text { for withdrawal among heterozygous patients. } \\
\text { Myelotoxicity and gastric intolerance occurred } \\
\text { significantly more frequently among heterozygous } \\
\text { than with TPMT wild-type patients }\end{array}$ \\
\hline $\begin{array}{l}\text { Newman et } \mathrm{al}^{16} \text {, } \\
\text { TARGET trial }\end{array}$ & $\begin{array}{l}\text { Individualized/ } \\
\text { prospective }\end{array}$ & $\begin{array}{l}\text { Arm I: Standard dosing } \\
\text { without genotyping vs } \\
\text { Arm 2: Pretreatment TPMT } \\
\text { genotyping and AZA dosing. } \\
\text { Wild-type ( } 1.5-3 \mathrm{mg} / \mathrm{kg} / \text { day); } \\
\text { heterozygous }(25-50 \mathrm{mg} / \text { day) } \\
\text { and titrate to the maintenance } \\
\text { dose; homozygous for TPMT } \\
\text { variant alleles were given } \\
\text { alternative drugs }\end{array}$ & $\begin{array}{l}\text { TPMT*2, *3A, } \\
* 3 B \text { and } * 3 C\end{array}$ & $\begin{array}{l}\text { No differences between the two study arms or } \\
\text { between heterozygous and wild-type homozygous } \\
\text { patients with respect to the rate of stopping } \\
\text { azathioprine due to ADRs. No difference in the rate } \\
\text { of remission between the intervention and control } \\
\text { groups }\end{array}$ \\
\hline $\begin{array}{l}\text { Coenen et } \mathrm{al}^{22} \text {, } \\
\text { TOPIC trial }\end{array}$ & $\begin{array}{l}\text { Individualized/ } \\
\text { prospective }\end{array}$ & $\begin{array}{l}\text { Arm I: Control group. No } \\
\text { genotyping + standard dosing: } \\
2-2.5 \mathrm{mg} / \mathrm{kg} / \text { day AZA or I-I.5 } \\
\mathrm{mg} / \mathrm{kg} / \text { day } 6-\mathrm{MP} \text {. } \\
\text { Arm 2: Intervention group. } \\
\text { Genotyping + individualized } \\
\text { dosing. TPMT wild-type: same } \\
\text { as control; heterozygous: } 50 \% \\
\text { of control and homozygous } \\
\text { variant: } 0 \%-10 \% \text { of control }\end{array}$ & $\begin{array}{l}\mathrm{TPMT}^{*} 2, * 3 A \\
\text { and } * 3 C\end{array}$ & $\begin{array}{l}\text { No significant overall impact of TPMT genotype- } \\
\text { guided dosing of thiopurines on treatment efficacy } \\
\text { or on the risk of hematologic ADRs (i.e., leukopenia } \\
\text { and thrombocytopenia) between the genotyped } \\
\text { and nongenotyped arms. Carriers of at least one } \\
\text { genetic variant in the pharmacogenetics arm had } \\
\text { a significant reduction in the risk of hematologic } \\
\text { ADRs compared with same group in the } \\
\text { conventional arm }\end{array}$ \\
\hline
\end{tabular}

Abbreviations: 6-MP, 6-mercaptopurine; 6-TGN, 6-thioguanine nucleotides; ADRs: adverse drug reactions; AZA, azathioprine; TPMT, thiopurine S-methyltransferase; TARGET, TPMT: Azathioprine Response to Genotyping and Enzyme Testing; TOPIC, Thiopurine response Optimization by Pharmacogenetic testing in Inflammatory bowel disease Clinics.

and control groups indicating that the adjustment did not affect treatment efficacy. However, the investigators did not provide a stratified analysis addressing the differences in outcomes according to genotype groups within each study arm or between the two arms, probably due to small sample size. Moreover, it is worth mentioning that a single patient with homozygous variant genotype in the study who was on the nongenotyping arm and subsequently received the full dose of AZA developed severe neutropenia, which underlines the importance of genetic testing to identify this group of patients. ${ }^{16}$

A larger and more recent prospective RCT, which involved 783 IBD patients, the Thiopurine response Optimization by
Pharmacogenetic testing in Inflammatory bowel disease Clinics (TOPIC) trial, similarly showed no significant overall impact of TPMT genotype-guided dosing of thiopurines on treatment efficacy or on the risk of hematologic ADRs (i.e., leukopenia and thrombocytopenia) between the genotyped and nongenotyped arms (frequency: $7.4 \%$ vs $7.9 \%$; relative risk: $0.93 ; 95 \%$ CI: $0.57-1.52$ ). The efficacy results of this study further advocate that a reduced thiopurine dose does not result in under-treatment. Moreover, a subgroup analysis of this study, which compared only carriers of TPMT variants between the two arms, revealed that the pharmacogenetic approach was able to significantly decrease the risk of hematologic ADRs by 10 -fold in carriers of at least one genetic 
variant (frequency: $2.6 \%$ vs $22.9 \%$; relative risk: $0.11 ; 95 \%$ CI: $0.01-0.85){ }^{22}$ The results of the secondary aim of this study excluded any significant association between TPMT genotypes and anemia, hepatotoxicity, pancreatitis, skin rash, GI and general malaise, which is consistent with the results of the aforementioned meta-analysis and other results in the literature. ${ }^{2,22,65}$ It also suggested that factors other than TPMT genotype play an important role in the development of thiopurine-induced ADRs. ${ }^{24}$

\section{Autoimmune disorders}

Autoimmune diseases are a group of heterogeneous conditions that involve a destructive attack against the host's tissues launched by a deregulated immune system as in SLE, RA, AIH and generalized-eczematous disorders. Thus, treatment strategies are usually based on the use of immunosuppressants that act by modifying the activity of the immune system. AZA is widely used as an immunosuppressive agent in autoimmune diseases but its use is limited by its ADRs. ${ }^{14}$ Similar to the meta-analysis that focused on IBD, another meta-analysis, which included 651 patients with autoimmune diseases coming from 11 studies demonstrated that overall ADRs and AZA-induced bone marrow toxicity are significantly associated with TPMT polymorphisms with OR of 3.12 (95\% CI: 1.48-6.56) and 3.76 (95\% CI: 1.97-7.17), respectively. The results remained significant in two analysis, one that grouped the homozygous and heterozygous carriers into one reduced-activity group and the other that focused on heterozygous carriers only. The study also showed a significant association with GI with OR of 6.43 (95\% CI: 2.04-20.25), but the authors suspect that the observed association might have been driven by a single study, since after excluding this study, the association was no longer significant with OR of 2.1 (95\% CI: 0.36-12.42). ${ }^{14}$ The study also excludes the association of TPMT polymorphisms with hepatotoxicity. The sub-analysis that examined the association with myelosuppression according to the type of disease found significant results in SLE, RA and AIH subgroups. They also concluded that the risk prediction of bone marrow toxicity and overall ADRs based on TPMT variant-positive genotypes has high specificity $(94.10 \%$ and $92.93 \%$, respectively) but at the expense of sensitivity ( $16.30 \%$ and $14.85 \%$, respectively). ${ }^{14}$ Furthermore, in a prospective study that investigated the impact of TPMT-activity guided AZA dosing on the treatment response in patients with atopic eczema, the investigators concluded that TPMT-based dosing was able to maintain the drug efficacy while reducing the predicted toxicity. ${ }^{70}$

\section{Cost-effectiveness}

Most of the above studies concluded that TPMT testing could lead to improved prescribing of thiopurines, which would ultimately result in an increased treatment efficacy and a reduction in the rate and intensity of ADRs.Nonetheless, the cost-effectiveness of such an intervention is still open to debate. Only a few studies have addressed the costeffectiveness of TPMT pharmacogenetics interventions. In an effort toward this evaluation, a case study examined the cost-effectiveness of prospective TPMT genotyping in children with ALL treated with thiopurines and suggested positive results manifested in financial savings and a gain in life-years in the most favorable settings of the sensitivity analysis. ${ }^{71}$ Similarly, another study established a model based on a theoretical IBD population treated with AZA and found that pretreatment screening for TPMT genotype would be cost-effective in avoiding patient mortality due to myelosuppression. ${ }^{29}$ However, data coming from RCTs do not necessarily support this conclusion as one perspective study found that such a technique incurred excessive cost associated with genotyping but did not predict AZA-induced toxicity in IBD patients. ${ }^{72}$ Nonetheless, these studies were too small and not adequately powered to answer this question. ${ }^{16}$ A systematic review came to the conclusion that screening for TPMT activity either by genotyping or phenotyping was a cost-effective strategy that can be used to reduce healthcare costs while improving clinical effectiveness. ${ }^{73}$ Another study aimed at the evaluation of the added-value of genetic screening of TPMT followed by dose adjustment of AZA prior to the initiation of treatment found that genetic-based dosing dominated the standard dosing strategy in patients with rheumatological disease by reducing the treatment cost and the frequency of AZA-induced side effects. ${ }^{74}$ In a more recent prospective economic evaluation that was conducted alongside the TARGET study, in which the study aim was to test the cost-effectiveness of the TPMT genotyping approach in autoimmune diseases, the researchers concluded that the genetic approach had up to $71 \%$ probability of being costeffective depending on the cost of the genetic test. The results, however, were not conclusive as the observed economic advantage in the intervention group owing to lower use of resources was accompanied by a slight (almost negligible) reduction in the quality of life. ${ }^{75}$

\section{Impact on clinical practice}

Over the past decade, TPMT enzyme testing has gained a lot of acceptance, as reflected by the rapid increase in the number of tests performed in clinical practice. ${ }^{16,76}$ This 
sudden increase was the inevitable result of multiple factors supporting this approach, which include the increase in the available knowledge about the role of TPMT in treatment outcome, the stronger recommendations coming from clinical guidelines like the CPIC and the wider accessibility to genetic testing (i.e., larger availability, reduced cost, faster turnaround of results and shorter interpretation time). ${ }^{1,16}$ This shift in clinical practice was evaluated in the TARGET study, which observed that the physicians did follow the recommendations coming from British clinical guidelines (e.g., British Association of Dermatologists Therapy and British Society for Rheumatology) for TPMT heterozygous patients and chose a lower initial dose of AZA for those patients but the investigators also noted that the physicians used overall lower starting doses for wild-type patients as well. ${ }^{16}$ This "safe" practice reflects the physicians' reservation regarding the sensitivity and specificity of this test, which stems from the fact that being homozygous carrier of TPMT wild-type, although predictive of a reduced risk of AZA-induced myelosuppression, does not completely eliminate the possibility. Indeed, it was mentioned earlier that a fraction of TPMT wild-type patients can still have intermediate TPMT-activity and that other factors play a role in the development of this ADR. ${ }^{16,33,36}$ Moreover, other side effects such as hepatotoxicity, pancreatitis, nausea and vomiting cannot be predicted by TPMT testing. ${ }^{1,14,65}$ The adoption of pretreatment TPMT screening seems to vary according to discipline, as reported by one survey, with $94 \%$ of dermatologists, $60 \%$ of gastroenterologists and only $47 \%$ rheumatologists requesting it. ${ }^{76}$ This could be related to the level of evidence available in the domain of practice and the strength of the recommendations of the respective guidelines and protocols used by each specialist (e.g., UK guidelines in dermatology and gastroenterology recommend genetic screening while ALL-2011 protocol mandates it). ${ }^{1,11,76}$ However, from an evidence-based perspective, and besides the universally accepted association with hematotoxicity, the recommendations for preemptive genetic testing still have some margin to evolve. Plus, even in well-established scenarios, like in the case of myelosuppression in TPMT-deficient patients, strong evidence is still lacking to support that the pharmacogenetic approach would result in a significantly better outcome. ${ }^{14,65}$

\section{TPMT in the new era of sequencing}

The influence of genetic polymorphisms in the TPMT gene on treatment outcome has been well-documented and replicated in many studies. However, studies have also concluded that the genetic-based screening for TPMT activity should be interpreted with caution, as the activity of the TPMT enzyme can be co-influenced by other factors, and the development of thiopurine-induced ADRs is a multifactorial event. ${ }^{14}$ For instance, most of the presented studies inferred TPMT activity by genotyping the most common nonfunctional TPMT alleles while results of a recent study that explored the sequencing data suggest that in certain populations, the inferred activity can be refined by incorporating the genotypes of other alleles. The study also identified a new variant in the TPMT gene, TPMT*38 (T514C), which had an allelic frequency of $0.11 \%$ and was predicted to be a damaging mutation. ${ }^{15}$ Moreover, as increasingly reported by different studies, genetic variants in other genes involved in thiopurines metabolism like ITPA, HGPRT and MTHFR as well as variants in genes independent of TPMT can influence thiopurines treatment outcome. ${ }^{14,20,25,41,70,77}$ For example, genome-wide association studies have identified variants in the PACSIN2 gene that influence TPMT activity and were linked to 6-MP-related gastrointestinal toxicity in children with ALL, whereas variants in the NUDT15 gene were associated with thiopurineinduced leukopenia. ${ }^{26,41}$ However, since a lot of genes have significant differences in the frequencies of polymorphisms across major ethnic groups, it is important to evaluate the genetic profiles of patients in a global frame that considers all of the genes involved in a specific pathway to better understand the impact of ethnic diversity on drug response. One particularly interesting example of the role of pharmacoethnicity is the case of NUDT15 in Japanese population, in whom polymorphisms of this gene were associated with higher risk of toxicity and were more frequent than TPMTdeficient variants. ${ }^{26}$ Studies also suggest that combining the effects of such polymorphisms with variants in TPMT gene could strengthen the predictive power of the risk of developing thiopurines-related toxicity. ${ }^{14,22}$ This should soon become feasible with the breakthrough advances in sequencing and genotyping techniques. Indeed, in a recent study that tested the sensitivity, specificity and predictive values of the imputation of TPMT-alleles, most values were over $90 \%$, indicating that imputation of TPMT alleles can be used as a screening method for individuals with high-risk of developing serious thiopurine-induced ADRs. ${ }^{46}$ Furthermore, nongenetic factors should be taken into consideration before thiopurine initiation as they can have a big influence on the outcome and might interfere with the genotype-guided dosing. ${ }^{14}$

\section{Conclusion}

In conclusion, although it is currently well established that TPMT polymorphisms can explain a certain portion of 
thiopurine-induced ADRs, particularly hematotoxicity, it is surely not capable of predicting all of them. Indeed, many studies $^{2,14,22,65}$ have found that certain ADRs were not associated with a reduced TPMT activity such as pancreatitis and hepatotoxicity. This holds true in the context of ALL, IBD and the different types of autoimmune disorders. What is clear so far is that TPMT-deficient genotypes (homozygous variant carriers and compound heterozygous), and to lesser extent, heterozygous patients are predisposed to thiopurineinduced severe hematotoxicity. ${ }^{1,47}$ However, other factors such as disease progression and co-medications can also modulate the risk of myelosuppression, regardless of the genotype. While TPMT-deficient patients will definitely benefit from dose reduction of thiopurines, the validity of this approach for heterozygous carriers is still arguable since studies have shown that not all of these patients are intolerant to thiopurine; in fact, $30 \%-60 \%$ of heterozygous patients do tolerate it. ${ }^{33,47}$ Moreover, depending on the treated condition and treatment protocol used, TPMT wild-type patients also exhibit higher risks of worse outcome such as hematologic relapse in ALL and treatment failure in IBD, which adds an extra layer of complexity to the already troublesome process of finding the best therapeutic regimen that would ensure maximum efficacy and minimum toxicity. ${ }^{47}$ Consequently, regular clinical testing and hematologic assessment remain the mainstay in the monitoring of thiopurine treatment while genetic testing adds the advantage of refining the initial dosing and patientstratification processes, as well as suggesting customized monitoring for certain patient groups. One nice example backed with strong clinical evidence is the abovementioned scenario of myelosuppression. Preemptive TPMT genetic screening and tailored thiopurine initial dosing followed by upward/downward titration and hematological monitoring to a target level of myelosuppression can be considered a costeffective approach which would allow the prevention and early detection of myelosuppression in this vulnerable population without compromising the efficacy of the treatment. ${ }^{47}$

\section{Perspective}

While the goal of personalized medicine in general, and pharmacogenetics in particular, is to deliver patient-tailored treatments that would ensure maximum efficacy with minimum toxicity, the studies presented in this review make the argument that this is not an easy task. There is a balance to consider between treatment benefits and ADRs, which is controlled by multiple factors. This being said, what we can be sure of, for now, is that the more we get to know about the impact of pharmacogenetics on the variability of treatment response, the better we will be able to control the outcome to the advantage of the patient. Moreover, most pharmacoeconomic analyses have indicated that screening for TPMT pharmacogenetics promises to be cost-effective. With the advent of next-generation sequencing and the many breakthroughs in bioinformatics, the cost of analyzing the entire human genome is bound to drop, which would allow for greater accessibility to genetic data and a larger understanding of how their interactions with each other and with other factors influence the treatment. In the meantime, it is very promising to see that most major institutions have already incorporated preemptive TPMT screening in their treatment protocols to enhance treatment outcome and the continuously emerging long-term data proving the utility of doing so, which should encourage other institutions to follow.

\section{Acknowledgment}

Rachid Abaji is a scholar of the Cole Foundation and the Network of Applied Medical Genetics and acknowledges the support of both organizations.

\section{Disclosure}

The authors report no conflicts of interest in this work.

\section{References}

1. Higgs JE, Payne K, Roberts C, Newman WG. Are patients with intermediate TPMT activity at increased risk of myelosuppression when taking thiopurine medications? Pharmacogenomics. 2010;11(2):177-188.

2. Dong XW, Zheng Q, Zhu MM, Tong JL, Ran ZH. Thiopurine S-methyltransferase polymorphisms and thiopurine toxicity in treatment of inflammatory bowel disease. World J Gastroenterol. 2010;16(25): 3187-3195.

3. Kotur N, Dokmanovic L, Janic D, et al. TPMT gene expression is increased during maintenance therapy in childhood acute lymphoblastic leukemia patients in a TPMT gene promoter variable number of tandem repeat-dependent manner. Pharmacogenomics. 2015;16(15):1701-1712.

4. Chouchana L, Narjoz C, Beaune P, Loriot MA, Roblin X. Review article: the benefits of pharmacogenetics for improving thiopurine therapy in inflammatory bowel disease. Aliment Pharmacol Ther. 2012;35(1):15-36.

5. Gisbert JP, Gomollon F. Thiopurine-induced myelotoxicity in patients with inflammatory bowel disease: a review. Am J Gastroenterol. 2008;103(7):1783-1800.

6. Fraser AG, Orchard TR, Jewell DP. The efficacy of azathioprine for the treatment of inflammatory bowel disease: a 30 year review. Gut. 2002;50(4):485-489.

7. Lennard L. The clinical pharmacology of 6-mercaptopurine. Eur J Clin Pharmacol. 1992;43(4):329-339.

8. Lennard L. TPMT in the treatment of Crohn's disease with azathioprine. Gut. 2002;51(2):143-146.

9. Chouchana L, Narjoz C, Roche D, et al. Interindividual variability in TPMT enzyme activity: 10 years of experience with thiopurinepharmacogenetics and therapeutic drug monitoring. Pharmacogenomics. 2014;15(6):745-757.

10. Relling MV, Hancock ML, Rivera GK, et al. Mercaptopurine therapy intolerance and heterozygosity at the thiopurine S-methyltransferase gene locus. J Natl Cancer Inst. 1999;91(23):2001-2008. 
11. Lennard L. Implementation of TPMT testing. Br J Clin Pharmacol. 2014;77(4):704-714.

12. Wang L, Pelleymounter L, Weinshilboum R, et al. Very important pharmacogene summary: thiopurine S-methyltransferase. Pharmacogenet Genomics. 2010;20(6):401-405.

13. Vora A, Mitchell CD, Lennard L, et al. Toxicity and efficacy of 6-thioguanine vs. 6-mercaptopurine in childhood lymphoblastic leukaemia: a randomised trial. Lancet. 2006;368(9544):1339-1348.

14. Liu YP, Xu HQ, Li M, et al. Association between thiopurine S-methyltransferase polymorphisms and azathioprine-induced adverse drug reactions in patients with autoimmune diseases: a meta-analysis. PLoS One. 2015;10(12): 0144234.

15. Kim HY, Lee SH, Lee MN, et al. Complete sequence-based screening of TPMT variants in the Korean population. Pharmacogenet Genomics. 2015;25(3):143-146.

16. Newman WG, Payne K, Tricker K, et al. A pragmatic randomized controlled trial of thiopurinemethyltransferase genotyping prior to azathioprine treatment: the TARGET study. Pharmacogenomics. 2011;12(6):815-826.

17. Evans WE, Horner M, Chu YQ, Kalwinsky D, Roberts WM. Altered mercaptopurine metabolism, toxic effects, and dosage requirement in a thiopurinemethyltransferase-deficient child with acute lymphocytic leukemia. J Pediatr. 1991;119(6):985-989.

18. Yates CR, Krynetski EY, Loennechen T, et al. Molecular diagnosis of thiopurine S-methyltransferase deficiency: genetic basis for azathioprine and mercaptopurine intolerance. Ann Intern Med. 1997;126(8):608-614.

19. Evans WE, Hon YY, Bomgaars L, et al. Preponderance of thiopurine S-methyltransferase deficiency and heterozygosity among patients intolerant to mercaptopurine or azathioprine. J Clin Oncol. 2001;19(8):2293-2301.

20. Karas-Kuzelicki N, Jazbec J, Milek M, Mlinaric-Rascan I. Heterozygosity at the TPMT gene locus, augmented by mutated MTHFR gene, predisposes to 6-MP related toxicities in childhood ALL patients. Leukemia. 2009;23(5):971-974.

21. Peregud-Pogorzelski J, Tetera-Rudnicka E, Kurzawski M, et al. Thiopurine S-methyltransferase (TPMT) polymorphisms in children with acute lymphoblastic leukemia, and the need for reduction or cessation of 6-mercaptopurine doses during maintenance therapy: the Polish multicenter analysis. Pediatr Blood Cancer. 2011;57(4):578-582.

22. Coenen MJ, de Jong DJ, van Marrewijk CJ, et al. Identification of patients with variants in TPMT and dose reduction reduces hematologic events during thiopurinetreatment of inflammatory bowel disease. Gastroenterology. 2015;149(4):907-917.e7.

23. Relling MV, Hancock ML, Boyett JM, Pui CH, Evans WE. Prognostic importance of 6-mercaptopurine dose intensity in acute lymphoblastic leukemia. Blood. 1999;93(9):2817-2823.

24. Colombel JF, Ferrari N, Debuysere H, et al. Genotypic analysis of thiopurine S-methyltransferase in patients with Crohn's disease and severe myelosuppression during azathioprine therapy. Gastroenterology. 2000;118(6):1025-1030.

25. Stocco G, Cheok MH, Crews KR, et al. Genetic polymorphism of inosine triphosphate pyrophosphatase is a determinant of mercaptopurine metabolism and toxicity during treatment for acute lymphoblastic leukemia. Clin Pharmacol Ther. 2009;85(2):164-172.

26. Moriyama T, Nishii R, Perez-Andreu V, et al. NUDT15 polymorphisms alterthiopurine metabolism and hematopoietic toxicity. Nat Genet. 2016;48(4):367-373.

27. Dubinsky MC, Yang H, Hassard PV, et al. 6-MP metabolite profiles provide a biochemical explanation for 6-MP resistance in patients with inflammatory bowel disease. Gastroenterology. 2002;122(4):904-915.

28. Ansari A, Hassan C, Duley J, et al. Thiopurinemethyltransferase activity and the use of azathioprine in inflammatory bowel disease. Aliment Pharmacol Ther. 2002;16(10):1743-1750.

29. Winter J, Walker A, Shapiro D, Gaffney D, Spooner RJ, Mills PR. Costeffectiveness of thiopurinemethyltransferase genotype screening in patients about to commence azathioprine therapy for treatment of inflammatory bowel disease. Aliment Pharmacol Ther. 2004;20(6):593-599.
30. Appell ML, Berg J, Duley J, et al. Nomenclature for alleles of the thiopurinemethyltransferase gene. Pharmacogenet Genomics. 2013;23(4):242-248.

31. Collie-Duguid ES, Pritchard SC, Powrie RH, et al. The frequency and distribution of thiopurinemethyltransferase alleles in Caucasian and Asian populations. Pharmacogenetics. 1999;9(1):37-42.

32. Katara P, Kuntal H. TPMT polymorphism: when shield becomes weakness. Interdiscip Sci. 2016;8(2):150-155.

33. Lennard L, Cartwright CS, Wade R, Vora A. Thiopurinemethyltransferase and treatment outcome in the UK acute lymphoblastic leukaemia trial ALL2003. Br J Haematol. 2015;170(4):550-558.

34. Winter JW, Gaffney D, Shapiro D, et al. Assessment of thiopurinemethyltransferase enzyme activity is superior to genotype in predicting myelosuppression following azathioprine therapy in patients with inflammatory bowel disease. Aliment Pharmacol Ther. 2007;25(9):1069-1077.

35. Hindorf U, Appell ML. Genotyping should be considered the primary choice for pre-treatment evaluation of thiopurinemethyltransferase function. J Crohns Colitis. 2012;6(6):655-659.

36. Schmiegelow K, Forestier E, Kristinsson J, et al. Thiopurinemethyltransferase activity is related to the risk of relapse of childhood acute lymphoblastic leukemia: results from the NOPHO ALL-92 study. Leukemia. 2009;23(3):557-564.

37. Schaeffeler E, Fischer C, Brockmeier D, et al. Comprehensive analysis of thiopurine S-methyltransferase phenotype-genotype correlation in a large population of German-Caucasians and identification of novel TPMT variants. Pharmacogenetics. 2004;14(7):407-417.

38. Schmiegelow K, Al-Modhwahi I, Andersen MK, et al. Methotrexate/ 6-mercaptopurine maintenance therapy influences the risk of a second malignant neoplasm after childhood acute lymphoblastic leukemia: results from the NOPHO ALL-92 study. Blood. 2009;113(24): 6077-6084.

39. Booth RA, Ansari MT, Loit E, et al. Assessment of thiopurine S-methyltransferase activity in patients prescribed thiopurines: a systematic review. Ann Intern Med. 2011;154(12):814-823, W-295-W-298.

40. Levinsen M, Rosthoj S, Nygaard U, et al. Myelotoxicity after high-dose methotrexate in childhood acute leukemia is influenced by 6-mercaptopurine dosing but not by intermediate thiopurinemethyltransferase activity. Cancer Chemother Pharmacol. 2015;75(1):59-66.

41.Tamm R, Magi R, Tremmel R, et al. Polymorphic variation in TPMT is the principal determinant of TPMT phenotype: a meta-analysis of three genomewide association studies. Clin Pharmacol Ther. Epub 2016 Oct 22.

42. Ford L, Prout C, Gaffney D, Berg J. Whose TPMT activity is it anyway? Ann Clin Biochem. 2004;41(Pt 6):498-500.

43. Lennard L, Chew TS, Lilleyman JS. Human thiopurinemethyltransferase activity varies with red blood cell age. Br J Clin Pharmacol. 2001;52(5):539-546.

44. Yan L, Zhang S, Eiff B, et al. Thiopurinemethyltransferase polymorphic tandem repeat: genotype-phenotype correlation analysis. Clin Pharmacol Ther. 2000;68(2):210-219.

45. Lau J, Ioannidis JP, Terrin N, Schmid CH, Olkin I. The case of the misleading funnel plot. BMJ. 2006;333(7568):597-600.

46. Almoguera B, Vazquez L, Connolly JJ, et al. Imputation of TPMT defective alleles for the identification of patients with high-risk phenotypes. Front Genet. 2014;5:96.

47. Relling MV, Gardner EE, Sandborn WJ, et al. Clinical pharmacogenetics implementation consortium guidelines for thiopurinemethyltransferase genotype and thiopurine dosing: 2013 update. Clin Pharmacol Ther. 2013;93(4):324-325.

48. Lennard L, Van Loon JA, Lilleyman JS, Weinshilboum RM. Thiopurinepharmacogenetics in leukemia: correlation of erythrocyte thiopurinemethyltransferase activity and 6-thioguanine nucleotide concentrations. Clin Pharmacol Ther. 1987;41(1):18-25.

49. Lennard L, Gibson BE, Nicole T, Lilleyman JS. Congenital thiopurinemethyltransferase deficiency and 6-mercaptopurine toxicity during treatment for acute lymphoblastic leukaemia. Arch Dis Child. 1993;69(5):577-579. 
50. Lennard L, Lilleyman JS, Van Loon J, Weinshilboum RM. Genetic variation in response to 6-mercaptopurine for childhood acute lymphoblastic leukaemia. Lancet. 1990;336(8709):225-229.

51. Dokmanovic L, Urosevic J, Janic D, et al. Analysis of thiopurine S-methyltransferase polymorphism in the population of Serbia and Montenegro and mercaptopurine therapy tolerance in childhood acute lymphoblastic leukemia. Ther Drug Monit. 2006;28(6):800-806.

52. Relling MV, Rubnitz JE, Rivera GK, et al. High incidence of secondary brain tumours after radiotherapy and antimetabolites. Lancet. 1999;354(9172):34-39.

53. Relling MV, Yanishevski Y, Nemec J, et al. Etoposide and antimetabolite pharmacology in patients who develop secondary acute myeloid leukemia. Leukemia. 1998;12(3):346-352.

54. Pui CH, Pei D, Sandlund JT, et al. Long-term results of St Jude Total Therapy Studies 11, 12, 13A, 13B, and 14 for childhood acute lymphoblastic leukemia. Leukemia. 2010;24(2):371-382.

55. Relling MV, Pui CH, Cheng C, Evans WE. Thiopurinemethyltransferase in acute lymphoblastic leukemia. Blood. 2006;107(2):843-844.

56. Rocha JC, Cheng C, Liu W, et al. Pharmacogenetics of outcome in children with acute lymphoblastic leukemia. Blood. 2005;105(12):4752-4758.

57. Moriyama T, Relling MV, Yang JJ. Inherited genetic variation in childhood acute lymphoblastic leukemia. Blood. 2015;125:3988-3995.

58. Schmiegelow K, Forestier E, Hellebostad M, et al. Long-term results of NOPHO ALL-92 and ALL-2000 studies of childhood acute lymphoblastic leukemia. Leukemia. 2010;24(2):345-354.

59. Schmiegelow K, Bjork O, Glomstein A, et al. Intensification of mercaptopurine/methotrexate maintenance chemotherapy may increase the risk of relapse for some children with acute lymphoblastic leukemia. J Clin Oncol. 2003;21(7):1332-1339.

60. Thomsen JB, Schrøder H, Kristinsson J, et al. Possible carcinogenic effect of 6-mercaptopurine on bone marrow stem cells. Cancer. 1999;86(6):1080-1086.

61. Levinsen M, Rotevatn EØ, Rosthøj S, et al. Pharmacogenetically based dosing of thiopurines in childhood acute lymphoblastic leukemia: influence on cure rates and risk of second cancer. Pediatric Blood Cancer. 2014;61(5):797-802.

62. Lennard L, Cartwright CS, Wade R, Vora A. Thiopurine dose intensity and treatment outcome in childhood lymphoblastic leukaemia: the influence of thiopurine methyltransferase pharmacogenetics. Br J Haematol. 2015;169(2):228-240.

63. Stanulla M, Schaeffeler E, Flohr T, et al. Thiopurine methyltransferase (TPMT) genotype and early treatment response to mercaptopurine in childhood acute lymphoblastic leukemia. JAMA. 2005;293(12):1485-1489.

64. Stanulla M, Schaeffeler E, Moricke A, et al. Thiopurine methyltransferase genetics is not a major risk factor for secondary malignant neoplasms after treatment of childhood acute lymphoblastic leukemia on Berlin-Frankfurt-Munster protocols. Blood. 2009;114(7):1314-1318.
65. Liu YP, Wu HY, Yang X, et al. Association between thiopurine S-methyltransferase polymorphisms and thiopurine-induced adverse drug reactions in patients with inflammatory bowel disease: a meta-analysis. PLoS One. 2015;10(3): 0121745.

66. de Jong DJ, Derijks LJ, Naber AH, Hooymans PM, Mulder CJ. Safety of thiopurines in the treatment of inflammatory bowel disease. Scand J Gastroenterol Suppl. 2003;(239):69-72.

67. Hindorf U, Lindqvist M, Peterson C, et al. Pharmacogenetics during standardised initiation of thiopurine treatment in inflammatory bowel disease. Gut. 2006;55(10):1423-1431.

68. Ansari A, Arenas M, Greenfield SM, et al. Prospective evaluation of the pharmacogenetics of azathioprine in the treatment of inflammatory bowel disease. Aliment Pharmacol Ther. 2008;28(8):973-983.

69. Derijks LJ, Gilissen LP, Engels LG, et al. Pharmacokinetics of 6-mercaptopurine in patients with inflammatory bowel disease: implications for therapy. Ther Drug Monit. 2004;26(3):311-318.

70. Meggitt SJ, Gray JC, Reynolds NJ. Azathioprine dosed by thiopurinemethyltransferase activity for moderate-to-severe atopic eczema: a double-blind, randomised controlled trial. Lancet. 2006;367(9513): 839-846.

71. van den Akker-van Marle ME, Gurwitz D, Detmar SB, et al. Costeffectiveness of pharmacogenomics in clinical practice: a case study of thiopurinemethyltransferase genotyping in acute lymphoblastic leukemia in Europe. Pharmacogenomics. 2006;7(5):783-792.

72. Sayani FA, Prosser C, Bailey RJ, Jacobs P, Fedorak RN. Thiopurine methyltransferase enzyme activity determination before treatment of inflammatory bowel disease with azathioprine: effect on cost and adverse events. Can J Gastroenterol. 2005;19(3):147-151.

73. Payne K, Newman WG, Gurwitz D, Ibarreta D, Phillips KA. TPMT testing in azathioprine: a 'cost-effective use of healthcare resources'? Personal Med. 2008;6(1):103-113.

74. Marra CA, Esdaile JM, Anis AH. Practical pharmacogenetics: the cost effectiveness of screening for thiopurine S-methyltransferase polymorphisms in patients with rheumatological conditions treated with azathioprine. J Rheumatol. 2002;29(12):2507-2512.

75. Thompson AJ, Newman WG, Elliott RA, Roberts SA, Tricker K, Payne K. The cost-effectiveness of a pharmacogenetic test: a trialbased evaluation of TPMT genotyping for azathioprine. Value Health. 2014;17(1):22-33.

76. Fargher EA, Tricker K, Newman W, et al. Current use of pharmacogenetic testing: a national survey of thiopurine methyltransferase testing prior to azathioprine prescription. J Clin Pharm Ther. 2007;32(2): 187-195.

77. Zelinkova Z, Derijks LJ, Stokkers PC, et al. Inosine triphosphate pyrophosphatase and thiopurine s-methyltransferase genotypes relationship to azathioprine-induced myelosuppression. Clin Gastroenterol Hepatol. 2006;4(1):44-49.
Pharmacogenomics and Personalized Medicine

\section{Publish your work in this journal}

Pharmacogenomics and Personalized Medicine is an international, peerreviewed, open access journal characterizing the influence of genotype on pharmacology leading to the development of personalized treatment programs and individualized drug selection for improved safety, efficacy and sustainability. This journal is indexed on the American Chemical

\section{Dovepress}

Society's Chemical Abstracts Service (CAS). The manuscript management system is completely online and includes a very quick and fair peer-review system, which is all easy to use. Visit http://www.dovepress. com/testimonials.php to read real quotes from published authors. 